\title{
Factors influencing adherence to referral advice following pre- referral treatment with artesunate suppositories in children in rural Tanzania
}

\author{
Daudi Simba \\ Muhimbili University of Health and Allied Sciences, Dar es Salaam, Tanzania \\ Marian Warsame \\ World Health Organization \\ Omari Kimbute \\ National Institute for Medical Research, Mtwara, Tanzania \\ Deodatus Kakoko \\ Muhimbili University of Health and Allied Sciences, Dar es Salaam, Tanzania \\ Max Petzold \\ Karolinska Institutet, Stockholm, Sweden
}

See next page for additional authors

Follow this and additional works at: https://ecommons.aku.edu/eastafrica_fhs_mc_pathol

Part of the Pathology Commons

\section{Recommended Citation}

Simba, D., Warsame, M., Kimbute, O., Kakoko, D., Petzold, M., Tomson, G., Premji, Z., Gomes, M. (2009).

Factors influencing adherence to referral advice following pre-referral treatment with artesunate suppositories in children in rural Tanzania. Tropical Medicine \& International Health, 14(7), 775-783.

Available at: https://ecommons.aku.edu/eastafrica_fhs_mc_pathol/63 


\section{Authors}

Daudi Simba, Marian Warsame, Omari Kimbute, Deodatus Kakoko, Max Petzold, Goran Tomson, Zul

Premji, and Melba Gomes 


\title{
Factors influencing adherence to referral advice following pre-referral treatment with artesunate suppositories in children in rural Tanzania
}

\author{
Daudi O. Simba ${ }^{1,2}$, Marian Warsame ${ }^{3}$, Omari Kimbute ${ }^{4}$, Deodatus Kakoko ${ }^{5}$, Max Petzold ${ }^{2,6}$, Goran Tomson ${ }^{2,7}$, \\ Zul Premji ${ }^{8}$ and Melba Gomes ${ }^{9}$ \\ 1 Department of Community Health, Muhimbili University of Health and Allied Sciences, Dar es Salaam, Tanzania \\ 2 Division of International Health (IHCAR), Department of Public Health Sciences, Karolinska Institutet, Stockbolm, Sweden \\ 3 Global Malaria Programme, World Health Organization, Geneva, Switzerland \\ 4 National Institute for Medical Research, Mtwara, Tanzania \\ 5 Department of Behavioural Sciences, Muhimbili University of Health and Allied Sciences, Dar es Salaam, Tanzania \\ 6 Nordic School of Public Health, Göteborg, Sweden \\ 7 Medical Management Centre, Karolinska Institutet, Stockholm, Sweden \\ 8 Department of Medical Parasitology and Entomology, Mubimbili University of Health and Allied Sciences, Dar es Salaam, Tanzania \\ 9 UNICEF/UNDP/World Bank/WHO Special Programme for Research and Training in Tropical Diseases (TDR) Geneva, Switzerland
}

Summary OBJECTIVE WHO recommends artemisinin suppository formulations as pre-referral treatment for children who are unable to take oral medication and cannot rapidly reach a facility for parenteral treatment. We investigated factors influencing caretakers' adherence to referral advice following pre-referral treatment of their children with rectal artesunate suppositories.

METHODS The study was nested within an intervention study that involved pre-referral treatment of all children who came to a community dispenser for treatment because they were unable to take oral medications because of repeated vomiting, lethargy, convulsions or altered consciousness. All patients who did not comply with referral advice were stratified by actions taken post-referral: taking their children to a drug shop, a traditional healer, or not seeking further treatment, and added to a random selection of patients who complied with referral advice. Caretakers of the children were interviewed about their socioeconomic status (SES), knowledge about malaria, referral advice given and actions they took following pre-referral treatment. Interview data for 587 caretakers were matched with symptoms of the children, the time of treatment, arrival at a health facility or other actions taken post-pre-referral treatment. RESULTS The majority (93.5\%) of caretakers reported being given referral advice by the community drug dispenser. The odds of adherence with this advice were three times greater for children with altered consciousness and/or convulsions than for children with other symptoms [odds ratio (OR) 3.47, 95\% confidence interval (CI) $2.32-5.17, P<0.001]$. When questioned, caretakers who remembered when (OR 2.19, 95\% CI 1.48-3.23, $P<0.001$ ) and why (OR 1.77, 95\% CI 1.07-2.95, $P=0.026$ ) they were advised to proceed to health facility - were more likely to follow referral advice. Cost did not influence adherence except within a catchment area of facilities that charged for services. In these areas, costs deterred adherence by four to five times for those who had previously paid for laboratory services $(\mathrm{OR}=0.25,95 \% \mathrm{CI}: 0.09-0.67, P=0.006)$ or consultation $(\mathrm{OR} 0.20,95 \% \mathrm{CI}: 0.06-0.61, P=0.005)$ compared with those who had not.

CONCLUSION When given referral advice, caretakers of patients with life-threatening symptoms adhere to referral advice more readily than other caretakers. Health service charges deter adherence.

keywords malaria, referral, artesunate, community based, rural

\section{Introduction}

In severe malaria, death occurs rapidly. Mortality is avoidable, and reflects delay in effective treatment (Barnes et al. 2004; White 2004; Dondorp et al. 2005), especially in African children who are at high risk (Snow \& Guerra 2005). Without successful control of uncomplicated malaria, a small proportion of patients will progress to 
D. O. Simba et al. Factors influencing adherence to referral advice

severe malaria requiring immediate effective parenteral antimalarial treatment. Risk of mortality is usually highest within the first $48 \mathrm{~h}$ after onset of severe symptoms, that is within one cycle of the blood stage infection. Many patients die either before reaching hospital (Breman 2001) or immediately thereafter (Dondorp et al. 2005). These deaths can be avoided by a single inexpensive artesunate suppository at the time of referral (Gomes et al. 2008). The artemisinin derivatives act rapidly and kill young parasites, thereby preventing their development to mature pathogenic stages (White 2004), a valuable characteristic in treating severe malaria. Current WHO Guidelines for the Treatment of Malaria recommend use of artemisinin-based suppositories as an emergency treatment prior to referral of the patient to health a facility for comprehensive management (WHO 2006). Adherence to referral advice is critical to the success of this strategy.

There are no data on routine compliance with referral advice or the factors that influence compliance with such advice after pre-referral treatment, although it has been reported that severity of the illness, need for permission to make the journey to clinic and bear associated costs, juggling child care responsibilities, perceived quality of hospital care and health workers' communication skills are important factors influencing adherence advice to proceed to hospitals (De Zoysa et al. 1998; Kalter et al. 2003). There are concerns as to whether and how many treated patients will follow referral advice if pre-referral treatment becomes routinely available at the community level: failure to adhere to referral advice might delay identification of the real cause of the disease while rapid recovery may lead to caretakers' perception that no further treatment is necessary (Hinton et al. 2007). This study was therefore undertaken to better understand the factors influencing parents' decisions to comply with advice to go to a health facility following pre-referral treatment, so that these factors could assist in designing appropriate health education materials to improve compliance.

\section{Methods and materials}

\section{Study area and population}

The study was conducted from June to August 2007 in Mtwara Rural District, southern Tanzania. The district has a total population of 212000 residing in 112 villages, of which 67 have either no health facility or are situated more than $5 \mathrm{~km}$ from a health facility, leading to delays in health care in life-threatening circumstances. The intervention was conducted in these 67 villages without immediate access to health care facilities. Malaria transmission is stable and high with falciparum malaria as the leading cause of outpatient attendances and hospital admissions, predominantly among children under-five (Warsame et al. 2007). The backbone of the district's health services is a network of health facility units at different levels: 30 dispensaries with outpatient services, four health centres with limited in-patient services and a regional hospital. In each village, there are two trained village health workers and several traditional birth attendants who deliver community-based health services.

The study was nested within a community-based intervention deploying artesunate suppositories as pre-referral treatment in children assessed as probably having malaria, and unable to take oral medication because of repeated vomiting, lethargy, altered consciousness or convulsions. Community drug dispensers comprised registered village health workers or about four selected mothers per village, who were trained to treat, refer all treated children to the nearest health facility for comprehensive management, follow-up the child at home post-treatment to establish outcome and document treatment-seeking practices after suppository treatment. Emphasis on the importance of compliance with advice to go to a health facility postpre-referral treatment was communicated through community educational posters prior to and during the intervention in which community drug dispensers treated patients, stressed immediate referral to health facilities in addition to providing a referral slip to the parents of the sick child.

\section{Study design and sample size}

Parents of children who did not comply with referral advice were identified from 2200 children treated in the intervention study, and grouped by their actions taken following pre-referral treatment: taking their children to drug stores, traditional healers or nowhere. The target was to have a minimum of 200 caretakers sampled by their post-pre-referral treatment actions. However, the target was not reached; if there were fewer than or approximately 200 children in a group, caretakers of all children in that stratum were selected; thus, all 52 caretakers who sought care from traditional healers, all 201 who did not seek definitive care for the respective episode and all 202 who went to purchase drugs from a drug shop were identified for interview. A further 286 of the 1810 caretakers who followed referral advice to go to a health facility were randomly selected as a comparison group, as well as 16 caretakers with no information on any action taken following pre-referral treatment. This gave us a sampling frame of 757 caretakers to be visited and interviewed to determine factors influencing adherence to referral advice. 
D. O. Simba et al. Factors influencing adherence to referral advice

\section{Study conduct}

Four research assistants were trained to administer a questionnaire on SES and actions taken after pre-referral treatment with an artesunate suppository; interviews were supervised by the principal investigator (DO Simba) and two associate researchers (D Kakoko and O Kimbute) throughout the conduct of the study. The caretakers who were present during the treated episode were interviewed at their homes after obtaining written informed consent. Where a caretaker who had been available at the time of the treated episode was not available for interview, a maximum of two additional visits were attempted.

Through a pre-tested structured questionnaire information on: (i) demographic status (age, sex, level of education and marital status), (ii) SES using 19 assets as proxies adopted from the demographic and health survey and the national census that included household assets (such as possession of radio, bicycle and livestock and other assets and household characteristics such as materials of the floor, walls and roof), (iii) knowledge about malaria (cause, transmission, prevention and appropriate treatment for malaria) and (iv) attitude of caretakers towards the quality of health care provided by health facilities (staff adequacy in number, availability of drugs, promptness of services and perceived adequacy of care) prompting caretakers to describe very poor, inadequate, undecided, adequate or very good services. Caretakers were also asked how much they normally paid for consultation, drugs and laboratory services at health facilities. To encourage neutral and unbiased questioning of caretakers, interviewers were not aware of whether caretakers had adhered with referral advice until data analysis.

Data generated through the interviews were matched with the following information on the index episode obtained at the time of treatment: presenting symptoms (inability to eat, drink or suck, repeated vomiting, lethargy, convulsions or altered consciousness), time of treatment categorized into morning (0500-1200 h), afternoon (1200-1600 h), evening (1600-1800 h) and night (1800$0500 \mathrm{~h}$ ), and actions taken after the child had been treated. Management of the child at any health facility after treatment defined adherence to referral advice; adherence was established by tracing the referral slips given to the parent at the time of treatment and other documentation at health facilities which confirmed whether the child had arrived at the facility or not; this procedure was successful for almost three-quarters (73.4\%: $210 / 286)$ of the patients arriving at the facility. We were not able to confirm adherence for the remainder (76/286 cases) because of inadequate record keeping in the referral health facilities. Non-adherence was defined as not going to a health facility but going to drug shops, to traditional healers or not consulting any health practitioners.

\section{Data analysis}

EPI-DATA software (EpiData Association, Odense, Denmark) was used for double data entry and validation. STATA versions 9 and 10 (Stata Corp., College Station, TX, USA) was used for analysis. Factors (independent variables) likely to influence adherence to referral advice (outcome variable) were analyzed using $2 \times 2$ tables. Strength of association was measured using chi-square tests; for $P$-values $<0.05$, the significance level is stated. Multiple logistic regression models were used to establish the independent effect on adherence (the dependent variable) of: demographic variables, clinical symptoms, caretakers' knowledge about cause of malaria and caretakers' memory of referral instructions given by drug dispenser. Because the sampling fraction for those who completed referral was different from the other strata, weighted regression analysis was applied in a pooled analysis. The measure of association selected was the odds ratio (OR) with $95 \%$ confidence intervals (CI).

\section{Ethical issues}

Ethical approval for the adherence study was obtained from the Muhimbili University College of Health Science and from the National Institute of Medical Research. Ethical review and approval for the intervention study was obtained from the National Institute of Medical Research and the World Health Organization Ethics Review Committee. Permission to conduct the study was obtained from the regional, district and village authorities. Individual informed consent was sought and obtained from the caretakers of the children in both the intervention and the nested adherence study.

\section{Results}

Of the 757 selected caretakers, $588(77.7 \%)$ were successfully located and interviewed but one child was removed from the analysis as the patient did not meet the indication for treatment. Of the remaining 587 caretakers, 96 had migrated to other areas and 71 could not be found at their homes after three visits to the household during the study period (Figure 1). Attrition was not apparently biased except for follow-up of patients who were taken to traditional healers: $77-81 \%$ successful follow-up overall with $81.0 \%(163 / 201)$ follow-up among patients who did not go anywhere; $78.7 \%$ (159/202) follow-up of patients who went to drug shops; $76.9 \%(220 / 286)$ follow-up of 
D. O. Simba et al. Factors influencing adherence to referral advice

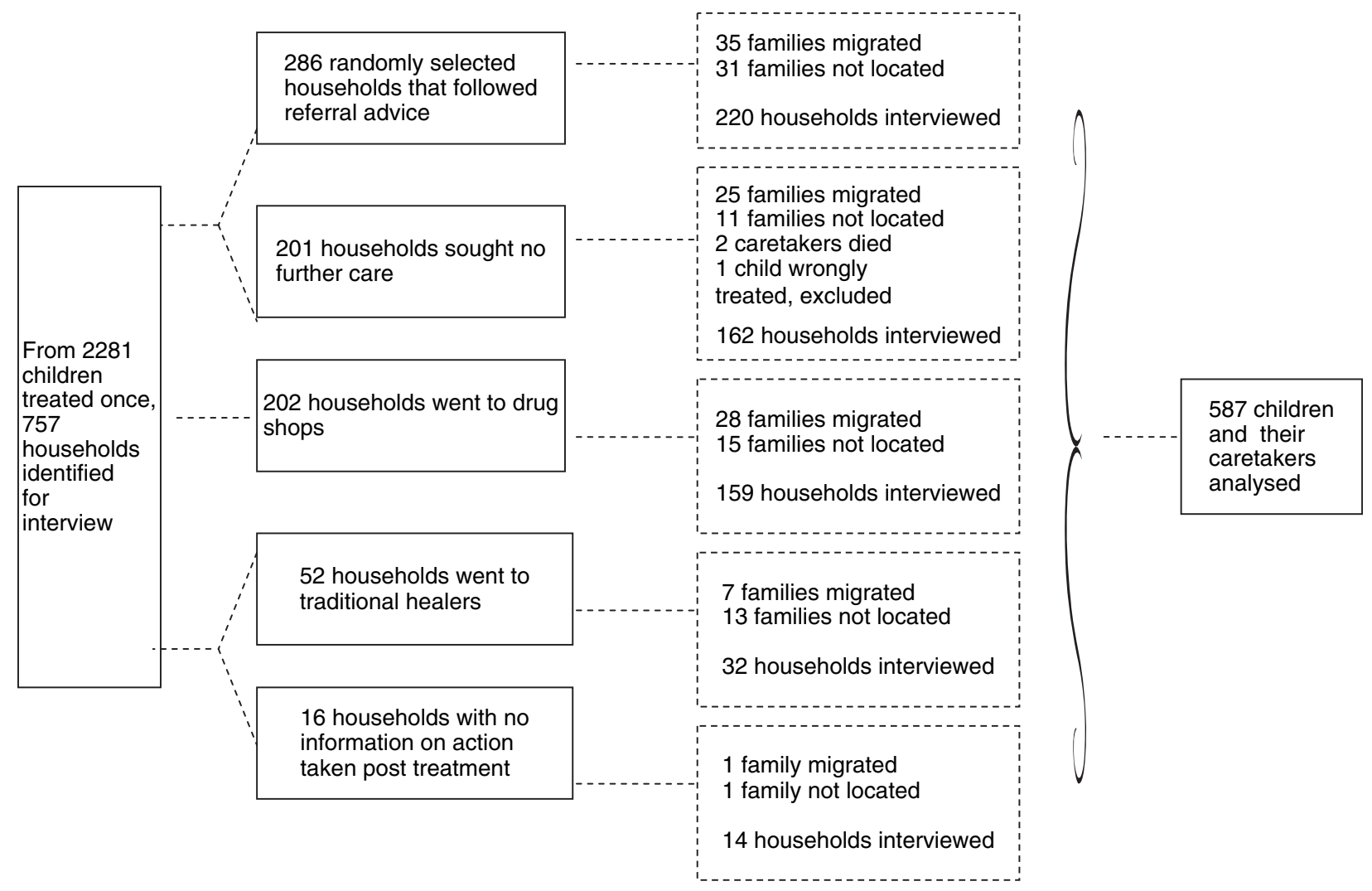

Figure I Flowchart. The number of children whose caretakers were interviewed and numbers not interviewed.

those who went to health facilities but $61.5 \%(32 / 52)$ follow-up among caretakers who went to traditional healers. There was no child in the sample who died.

Table 1 summarizes the socio-demographic profile of the caretakers, presenting symptoms of their children at the time of pre-referral treatment and adherence by caretakers to referral advice. There was no significant difference in adherence by caretakers' age, level of education and marital status. Almost one-third $(30.8 \%)$ of the children presented with altered consciousness and/or repeated convulsions and two-thirds $(68.5 \%)$ had repeated vomiting, lethargy and/or were unable to eat/drink/suck (Table 1). The majority of caretakers of children with altered consciousness and/or convulsions adhered to advice to proceed to health facility compared with parents of patients with other symptoms $(P<0.0001$, Table 1$)$. There was no significant difference in the actions taken by gender of the child.

Table 2 presents actions taken by caretakers following pre-referral treatment and symptoms of their children at presentation. More patients with relatively serious symptoms, such as altered consciousness and/or convulsions, went to a health centre; fewer of these patients went to a drug shop and there was no indication that these patients were systematically taken to a traditional healer (Table 2).

Table 3 provides the results of multiple logistics regression analysis assessing factors that independently influenced adherence; the pooled analysis gives the weighted results. Variables introduced into the model included demographic and socio-economic background of caretakers, clinical symptoms of the children, time at which prereferral treatment was sought and caretakers' recollection of instructions regarding referral advice. The odds of adhering to referral advice to go to a health facility was three times greater for patients with altered consciousness and/or convulsions as compared with children with the other symptoms (OR 3.47, 95\% CI 2.52-5.17, $P<0.0001$ ), confirming the results of the test of proportions and signifying that the results were not diluted by the introduction of other independent variables into the regression model. Factors that were not significant at the 0.05 level were education, age, marital and SES of the caretakers and time of the day at which treatment was sought.

Most caretakers reported that they had been instructed to go to a health facility for further management of their 
D. O. Simba et al. Factors influencing adherence to referral advice

Table I Characteristics of caretakers interviewed and children's symptoms by adherence status

\begin{tabular}{|c|c|c|c|c|c|c|c|}
\hline \multirow{2}{*}{$\begin{array}{l}\text { Characteristics of } \\
\text { parent and child }\end{array}$} & \multirow{2}{*}{$\frac{\text { All patients }}{\text { No. }}$} & \multicolumn{2}{|c|}{$\begin{array}{l}\text { Adhered to } \\
\text { referral advice }\end{array}$} & \multicolumn{2}{|c|}{$\begin{array}{l}\text { Did not adhere } \\
\text { to referral advice }\end{array}$} & \multirow[b]{2}{*}{ Chi-square } & \multirow[b]{2}{*}{$P$-value } \\
\hline & & $n$ & $\%$ & $n$ & $\%$ & & \\
\hline \multicolumn{8}{|l|}{ Marital status } \\
\hline Unmarried & 112 & 39 & 34.8 & 73 & 65.2 & 0.4171 & 0.518 \\
\hline Married & 475 & 181 & 38.1 & 294 & 61.9 & & \\
\hline Total & 587 & 220 & 37.5 & 367 & 62.5 & & \\
\hline \multicolumn{8}{|l|}{ Caretaker's age } \\
\hline $15-24$ & 87 & 39 & 44.8 & 48 & 55.2 & 3.1007 & 0.376 \\
\hline $25-35$ & 231 & 80 & 34.6 & 151 & 65.4 & & \\
\hline $35-44$ & 175 & 68 & 38.9 & 107 & 61.1 & & \\
\hline $45+$ & 93 & 33 & 35.5 & 60 & 64.5 & & \\
\hline Total & $586 \dagger$ & 220 & 37.5 & 366 & 62.5 & & \\
\hline \multicolumn{8}{|l|}{ Caretaker's education } \\
\hline Below 7 years & 282 & 117 & 41.5 & 165 & 58.5 & 3.4740 & 0.062 \\
\hline 7 years and above & 300 & 102 & 34.0 & 198 & 66.0 & & \\
\hline Total & $582 \ddagger$ & 219 & 37.6 & 363 & 62.4 & & \\
\hline \multicolumn{8}{|c|}{ Child's symptoms at treatment } \\
\hline $\begin{array}{l}\text { Altered } \\
\text { consciousness/convulsions }\end{array}$ & 181 & 102 & 56.4 & 79 & 43.6 & 43.4 & 0.000 \\
\hline $\begin{array}{l}\text { Repeated vomiting, } \\
\text { unable to eat, drink or } \\
\text { suck, lethargic }\end{array}$ & 402 & 115 & 28.6 & 287 & 71.4 & & \\
\hline Not known & 4 & 3 & 75.0 & 1 & 25.0 & & \\
\hline Total & 587 & 220 & 37.5 & 367 & 62.5 & & \\
\hline
\end{tabular}

$\dagger$ Age missing for one caretaker.

\$Level of education missing for five caretakers.

Table 2 Actions taken by caretakers after treatment, by gender and clinical status of the treated child

\begin{tabular}{|c|c|c|c|c|c|c|c|c|}
\hline Action taken after treatment & & No. & $\%$ & Male no. & $\%$ & \multicolumn{2}{|l|}{ Female no. } & $\%$ \\
\hline Went to hospital/health centre & & 220 & 37.5 & 116 & 52.7 & 104 & & 47.3 \\
\hline Went to a drug shop & & 159 & 27.0 & 74 & 46.5 & 85 & & 53.5 \\
\hline Went to a traditional healer & & 32 & 5.5 & 16 & 50.0 & 16 & & 50.0 \\
\hline Did not take any action & & 162 & 27.6 & 86 & 53.1 & 76 & & 46.9 \\
\hline Not known & & 14 & 2.4 & 11 & 78.6 & 3 & & 21.4 \\
\hline Total & & 587 & 100 & 303 & 51.6 & 284 & & 48.4 \\
\hline $\begin{array}{l}\text { Action taken by clinical } \\
\text { status of child }\end{array}$ & No. & $\%$ & $\begin{array}{l}\text { Altered } \\
\text { consciousness } \\
\text { or convulsions }\end{array}$ & $\%$ & $\begin{array}{l}\text { Lethargy, vomiting, } \\
\text { unable to eat, drink } \\
\text { or suck }\end{array}$ & $\%$ & $\begin{array}{l}\text { Not } \\
\text { known }\end{array}$ & $\%$ \\
\hline Went to hospital/health centre & 220 & 37.5 & 102 & 46.4 & 115 & 52.3 & 3 & 1.3 \\
\hline Went to a drug shop & 159 & 27.1 & 32 & 20.8 & 126 & 79.2 & 0 & 0.0 \\
\hline Went to a traditional healer & 32 & 5.5 & 13 & 40.6 & 19 & 59.4 & 0 & 0.0 \\
\hline Did not take any action & 162 & 27.6 & 31 & 19.1 & 130 & 80.3 & 1 & 0.6 \\
\hline Not known & 14 & 2.3 & 2 & 14.3 & 12 & 85.7 & 0 & 0.0 \\
\hline Total & 587 & 100 & 181 & 30.8 & 402 & 68.5 & 4 & 0.7 \\
\hline
\end{tabular}

children $(93.5 \% ; 549 / 587)$ and of these $39.0 \%(214 / 549)$ were in the stratum that complied with the referral advice. Caretakers who remembered when to go to a health facility were twice as likely to comply with referral advice (OR $2.19,95 \%$ CI $1.48-3.23, P<0.001)$. If they remembered why they were instructed to go to the health facility they 
D. O. Simba et al. Factors influencing adherence to referral advice

Table 3 Factors affecting adherence to referral advice, with adjusted odds ratio (OR) from logistical regression analysis $(n=587)$

\begin{tabular}{|c|c|c|c|}
\hline & OR & $95 \% \mathrm{CI}$ & $P$-value \\
\hline \multicolumn{4}{|c|}{ Health facility $v s$ all actions (pooled results)* } \\
\hline Presenting more severe symptoms $\dagger$ & 3.47 & $2.32-5.17$ & 0.000 \\
\hline Knowledge about cause & 0.45 & $0.30-0.69$ & 0.000 \\
\hline Advice given about when to refer & 2.19 & $1.48-3.23$ & 0.000 \\
\hline Advice given why referring & 1.77 & $1.07-2.95$ & 0.026 \\
\hline \multicolumn{4}{|l|}{ Health facility $v s$ traditional healers } \\
\hline Presenting more severe symptoms $\dagger$ & 1.53 & $0.73-3.19$ & 0.259 \\
\hline Have knowledge about cause & 0.99 & $0.45-2.17$ & 0.983 \\
\hline Advice given about when to refer & 1.95 & $0.91-4.22$ & 0.088 \\
\hline Advice given why referring & 0.18 & $0.02-1.41$ & 0.103 \\
\hline \multicolumn{4}{|l|}{ Health facility $v s$ drug shops } \\
\hline Presenting more severe symptoms $\dagger$ & 3.25 & $2.02-5.25$ & 0.000 \\
\hline Have knowledge about cause & 0.44 & $0.27-0.70$ & 0.001 \\
\hline Advice given about when to refer & 1.59 & $1.02-2.48$ & 0.041 \\
\hline Advice given why referring & 2.10 & $1.21-3.67$ & 0.009 \\
\hline \multicolumn{4}{|l|}{ Health facilities $v s$ no action } \\
\hline Presenting more severe symptoms $\dagger$ & 4.00 & $2.43-6.60$ & 0.000 \\
\hline Have knowledge about cause & 0.52 & $0.32-0.83$ & 0.006 \\
\hline Advice given about when to refer & 3.05 & $1.94-4.79$ & 0.000 \\
\hline
\end{tabular}

*Pooled analysis done weighting by action taken.

$\dagger$ Symptoms at treatment were inability to eat, drink or suck, repeated vomiting, lethargy, repeated convulsions or altered consciousness. The latter two symptoms were classified as 'more severe' in the regression analysis.

were 1.8 times more likely to adhere (OR $1.77,95 \%$ CI 1.07-2.95, $P=0.026$ ) than those who remembered that they had been given referral advice but did not remember the content of the advice given.

Table 3 presents the odds of adherence within each of the actions taken by caretakers following referral advice. Severity of symptoms at the time of treatment and caretakers' memory of the referral advice given (when to go to the clinic and why) continued to be significant predictors of adherence in the bealth facility-drug shop comparison and in the bealth facility-no action comparison. In the bealth facility-traditional healer comparison, there were no variables introduced in the model, whether demographic, socio-economic or clinical symptoms of the child that contributed to an explanation of non-adherence and this is likely to be a result of the small numbers involved in this sub-group (Table 3).

About half of the caretakers, 56.1\% (329/587) reported paying for drugs. However, only a minority, $13.6 \%$ $(80 / 587)$ reported paying for laboratory services or for consultation with a health professional: $21.1 \%(124 / 587)$. Payment for services was clustered in 11 villages served by a health facility that required payment. In these villages, many caretakers reported paying for health services. For them, the odds of adhering to referral advice were four times lower (in the bealth facility-drug shop comparison and health facility-no action) and five times lower (in the bealth facility-no action comparison) than those who did not pay. There was no indication that severity of symptoms influenced adherence to referral advice. In villages served by public health facilities, payment for health care was rare for children under the age of 5 and consequently did not deter adherence to referral advice.

There was no apparent association between the perceived quality of care from the health facility, distance to facility, means of transport, SES of the caretaker and adherence to referral advice. However, caretakers who reported that they did not know the cause of malaria transmission had higher odds of adhering to referral advice (Table 3). We were unable to establish why this was the case.

\section{Discussion}

In remote rural communities without immediate access to parenteral medicines, any potential of pre-referral artesunate suppositories to halt disease progression and save life can only be realized if patients are given pre-referral treatment early and caretakers proceed with the patient to a clinic for assessment and management. Our results provide strong evidence that symptoms associated with cerebral malaria, which carries a high risk of death, or residual neurological damage in survivors (Molyneux 1989; Newton \& Krishna 1998; Carter et al. 2005; Ngoungou et al. 2006), were the most important factors influencing adherence to referral advice given by 
D. O. Simba et al. Factors influencing adherence to referral advice

community drug dispensers. There was no apparent preference to seek traditional care among children with convulsions or altered consciousness.

It has previously been reported from qualitative studies that convulsing children in Tanzania and elsewhere are either taken first or predominantly to traditional healers for the management of convulsions, even when they prefer modern care; when the moment of crisis is over or when their expectations of rapid cure are not met, they proceed to health facilities (Comoro et al. 2003; De Savigny et al. 2004; Kaona \& Tuba 2005; Okeke et al. 2006; Warsame et al. 2007). However, quantitative data on behaviour in fatal cases show a preference for modern care (De Savigny et al. 2004). Although we do not know whether parents chose treatment with rectal artesunate after a prior consultation at traditional care, once they sought rectal artesunate treatment, parents of children that were convulsing or had altered consciousness readily followed modern care and remembered referral advice given. Our study contributes to the literature that suggests that central nervous symptoms and referral advice can trigger modern (and in a minority of cases, traditional) care. Adherence was appropriate and decisive once felt to be necessary, since the time of day or night at which the child presented with these symptoms, distance to clinic, cost, gender, perceived quality of care at referral facility, literacy or SES did not interfere with adherence to referral advice for children with these symptoms. Indeed, caretakers who complied with referral advice had a clear memory of the advice that they had been given as to when and why they were advised to go health facility.

The results corroborate other findings that caretakers follow through on referrals to hospital when their child is critically ill and when they have been instructed to do so (De Zoysa et al. 1998; Muller et al. 2003). This implies that effective communication during provider-client interaction improves adherence (Winch et al. 2003; Chinbuah et al. 2006). Caretakers who remembered the referral advice given and recalled the reasons why they had been told to go to health facility had a higher chance of going there. This might argue strongly for a policy of training and supporting providers to treat, refer and effectively com municate to parents the reasons for rapid transfer to a hospital. There may be a need to reinforce referral advice in children with other danger signs - repeated vomiting, inability to take oral medication and lethargy.

Cost of treatment deterred adherence in the catchment area of a mission facility where payment for drugs, laboratory services or medical consultation was required. Although mission health facilities often charge less than private, for-profit, health facilities, the costs, even if small, were a deterrent for some caretakers in a society where nearly $40 \%$ of the population live below the poverty line (National Bureau of Statistics 2002). Cost did not influence adherence elsewhere in the study area. This is probably because the health system in Tanzania, unlike other countries where cost of treatment has been reported as a major factor influencing the choice of care for severe illness, exempts young children from user fees (De Zoysa et al. 1998; White 1998; Kalter et al. 2003; Peterson et al. 2004).

The lack of association between adherence to referral advice and geographic or socio-economic factors and a very high correlation between adherence, symptoms and the nature of the advice given is unusual. In many other studies, geographic and socio-economic factors were directly associated with adherence to referral advice (De Zoysa et al. 1998; Kalter et al. 2003; Macintyre et al. 2003; Kallander et al. 2006). One explanation is a lack of variation in SES in our study area compared with other areas studied. Secondly, the drug dispensers were trained to advise caretakers on the need for immediate transfer to the nearest health facility and although we had no means of establishing whether such advice was given, $98 \%$ of the respondents stated that they were referred to the nearest clinic. This high referral rate probably made the group treated more homogeneous. Furthermore, our study involved a narrow group of children, all of whom had danger signs and were treated with artesunate; other studies reported caretaker behaviour in circumstances where children had both urgent and non-urgent conditions (De Zoysa et al. 1998; Kallander et al. 2006) or caretakers were assessing referral from primary health facilities to hospitals (Kalter et al. 2003; Macintyre et al. 2003).

Follow-up therapy and management obtained by the treated children was not the subject of this study. Monotherapy with pre-referral artesunate suppositories for children with malaria which is not followed up by a combination curative therapy brings increased risk of drug resistance (McCombie 1996; Ansah et al. 2001; Hinton et al. 2007), as it is probable that the treated group will be hyperparasitaemic. Although parenteral artemisinins are also monotherapy, they are normally given under controlled conditions at hospitals where consolidation therapy can be initiated before discharge. In a community setting, the risk of using rectal artesunate must be weighed against the risk of death from severe malaria but should be followed by oral artemisinin combination therapy as soon as the child can take oral treatment reliably.

The weakest link in seeking effective care is likely to be in traditional societies in which traditional care for convulsions and altered consciousness may be the first resort or where switching between traditional and modern 
D. O. Simba et al. Factors influencing adherence to referral advice

care, or using only the former, will delay effective treatment. It may even be worthwhile exploring whether traditional healers can contribute in the provision of prereferral artesunate treatment and immediate referral of patients to hospital.

\section{Methodological considerations}

Symptoms, time of treatment and arrival at a health facility were obtained from a case record form filled out by the treatment provider at the time of treatment at the respective health facility. All other findings are reported by the caretakers, so there could be variations in recall when there was a long time lag between the index episode and the interview. Given the seriousness of the episode and the unusual treatment provided, we believe it is likely that caretakers remembered the circumstances surrounding the provision of treatment in the acute phase. Loss-to-followup was highest in the group that went to traditional healers post-treatment. This and the small number of those who went to traditional healers weakens the analysis of this group.

Although we had 19 variables as proxies for SES, we observed during analysis that many variables had a skewed outcome, that is $90 \%$ or more of the respondents were found in one category, reflecting a homogeneous SES in the sampled households. Of the variables that had a larger degree of variance, only roofing materials had any statistical relationship to adherence. The lack of variation in SES limited exploration of its influence upon adherence to referral advice.

\section{Conclusion}

Caretakers of patients with convulsions and/or altered consciousness who are asked to go to the nearest health facility after pre-referral treatment do so readily and remember the reasons why they were asked to go there. This reassuring finding suggests that, with adequate training of providers to give referral advice, caretakers of patients with a high absolute risk of death tend to access a health facility at which appropriate diagnosis and complete treatment can be provided.

\section{Acknowledgements}

We thank the Muhimbili University College of Health Sciences (MUHAS)/Karolinska Institutet (KI) collaboration for supporting the research and sub-study that led to this paper. The intervention 'deployment' study was supported by the UNICEF/UNDP/World Bank/WHO Special Programme for Research and Training in Tropical
Diseases. We convey special thanks to Annika Janson for her helpful advice and comments in the design of this study and to Mr Jan Singlovic for sampling the households for the sub-study and providing matched data from the deployment study, on symptoms, time of treatment and adherence. Our gratitude also goes to Prof Massele for the coordination of the MUHAS/KI collaboration through which this study was conducted. Finally, we express our gratitude to all participants, research assistants and others involved in support of the logistics arrangements for conducting of this study. The study on which this paper is based was funded by SIDA/SAREC through the MUHAS/KI - Malaria Project.

\section{References}

Ansah E, Gyapong J, Agyepong I \& Evans D (2001) improving adherence to malaria treatment for children: the use of prepacked chloroquine tablets vs chloroquine syrup. Tropical Medicine and International Health 6, 496-401.

Barnes K, Mwenechanya J, Tembo M et al. (2004) Efficacy of rectal artesunate compared with parenteral quinine in initial treatment of moderately severe malaria in African children and adults: a randomized study. Lancet 363, 1598-1605.

Breman J (2001) The ears of hippopotamus: manifestations, determinants, and estimates of the malaria burden. American Journal of Tropical Medicine and Hygiene 64, 1-11.

Carter J, Mung'ala-Odera V \& Neville B (2005) Persistent neurocognitive impairments associated with severe falciparum malaria in Kenyan children. Journal of Neurology, Neurosurgery, and Psychiatry 76, 476-481.

Chinbuah A, Gyapong J, Pagnoni F, Wellington E \& Gyapong M (2006) Feasibility and acceptability of the use of artemetherlumefantrine in the home management of uncomplicated malaria in children 6-59 months old in Ghana. Tropical Medicine and International Health 11, 1003-1016.

Comoro C, Nsimba SE, Warsame M \& Tomson G (2003) Local understanding, perceptions and reported practices of mothers/ guardians and health workers on childhood malaria in a Tanzanian district - implications for malaria control. Acta Tropica 87, 305-313.

De Savigny D, Mayombana C, Mwageni E et al. (2004) Careseeking patterns for fatal malaria in Tanzania. Malaria Journal $3,27$.

De Zoysa I, Bhandari N, Akhtari N et al. (1998) Careseeking for illness in young infants in an urban slum in India. Social Science and Medicine 47, 2101-2114.

Dondorp AN, Stepniewska F, Day N \& White NJ (2005) Artesunate versus quinine for treatment of severe falciparum malaria: a randomised trial. Lancet 366, 717-725.

Gomes M, Faiz MA, Gyapong JO et al. (2008) Pre-referral rectal artesunate to prevent death and disability in severe malaria: a placebo-controlled trial. Lancet 373, 557-566.

Hinton R, Auwun A, Pongua G et al. (2007) Caregivers' acceptance of using artesunate suppositories for treating child malaria 
D. O. Simba et al. Factors influencing adherence to referral advice

in papua new guinea. The American Journal of Tropical Medine and Hygiene 76, 634-640.

Kallander K, Tomson G, Nsungwa-Sabiiti J, Senyonjo Y, Pariyo G \& Petersen S (2006) Community referral in home management of malaria in Western Uganda: a case series study. BMC International Health and Human Rights 6, 2.

Kalter H, Salgado R, Moulton L et al. (2003) Factors constraining adherence to referral advice for severely ill children managed by the integrated management of childhood illness approach in Imbabura Province, Ecuador. Acta Paediatrica 92, 103-110.

Kaona F \& Tuba M (2005) A qualitative study to identify community structures for management of severe malaria: a basis for introducing rectal artesunate in the under five years children in Nakonde District of Zambia. BMC Public Health 5, 28.

Macintyre K, Lochigan M \& Letipila F (2003) Understanding referral from primary care clinics in rural Kenya: using health information systems to prioritize health services. The International Journal of Health Planning and Management 18, 2339.

McCombie S (1996) Treatment seeking for malaria - a review of recent research. Social Science and Medicine 43, 933-945.

Molyneux M (1989) Clinical features and prognostic indicators in paediatric cerebral malaria: a study of 131 comatose Malawian children. The Quarterly Journal of Medicine 71, 441-459.

Muller O, Traore C, Becher H \& Kouyata B (2003) Malaria morbidity, treatment seeking behaviour, and mortality in a cohort of young children in Burkina Faso. Tropical Medicine and International Health 8, 290-296.

National Bureau of Statistics (2002) Household Budget Survey in President's Office. National Bureau of Statistics, Tanzania.
Newton C \& Krishna S (1998) Severe falciparum malaria in children: current understanding of pathophysiology and supportive treatment. Pharmacology and Therapeutics 79, 1-53.

Ngoungou E, Dulac O \& Poudiougou B (2006) Epilepsy as a consequence of cerebral malaria in area in which malaria is endemic in Mali, West Africa. Epilepsia 47, 873-879.

Okeke T, Okafor H \& Uzochukwu B (2006) Traditional healers in Nigeria: perception of cause, treatment and referral practices for severe malaria. Journal of Biosocial Science 38, 491-500.

Peterson S, Nsungwa-Sabiiti J, Were W et al. (2004) Coping with paediatric referral - Ugandan parents' experience. Lancet 363 , 1955-1956.

Snow RW \& Guerra CA (2005) The global distribution of clinical episodes of Plasmodium falciparum malaria. Nature 434, 214217.

Warsame M, Kimbute O, Machinda Z et al. (2007) Recognition, perceptions and treatment practices for severe malaria in rural Tanzania: implications for accessing rectal artesunate as a prereferral. PLOS ONE 2, e149.

White $\mathrm{N}$ (1998) Why is it that antimalarial drug treatments do not always work? Annals of Tropical Medicine and Parasitology 92 , 367-391.

White NJ (2004) Antimalarial drug resistance. The Journal of Clinical Investigation 113, 1084-1092.

WHO (2006) Guidelines for the Treatment of Malaria. World Health Organisation, Geneva.

Winch P, Bagayoko A, Diawar A et al. (2003) Increases in correct administration of chloroquine in the home and referral of sick children to health facilities through a community-based intervention in Bougouni District, Mali. Transactions of the Royal Society of Tropical Medicine and Hygiene 97, 481-490.

Corresponding Author Daudi O. Simba, Department of Community Health, Muhimbili University of Health and Allied Sciences, Dar es Salaam, Tanzania. E-mail: dsimba@muhas.ac.tz 